MPP-2013-214

\title{
Neutrino flavor pendulum in both mass hierarchies
}

\author{
Georg Raffelt and David de Sousa Seixas \\ Max-Planck-Institut für Physik (Werner-Heisenberg-Institut), Föhringer Ring 6, 80805 München, Germany
}

(Dated: 29 July 2013, updated 22 August 2013)

\begin{abstract}
We construct a simple example for self-induced flavor conversion in dense neutrino gases showing new solutions that violate the symmetries of initial conditions. Our system consists of two opposite momentum modes 1 and 2, each initially occupied with equal densities of $\nu_{e}$ and $\bar{\nu}_{e}$. Restricting solutions to symmetry under $1 \leftrightarrow 2$ allows for the usual bimodal instability ("flavor pendulum") in the inverted neutrino mass hierarchy (IH) and stability (no self-induced flavor conversion) in the normal hierarchy $(\mathrm{NH})$. Lifting this symmetry restriction allows for a second pendulumlike solution that occurs in NH where the modes 1 and 2 swing in opposite directions in flavor space. Any small deviation from 1-2 symmetry in the initial condition triggers the new instability in NH. This effect corresponds to the recently identified multi-azimuth angle (MAA) instability of supernova neutrino fluxes. Both cases show explicitly that solutions of the equations of collective flavor oscillations need not inherit the symmetries of initial conditions, although this has been universally assumed.
\end{abstract}

PACS numbers: 14.60.Pq, 97.60.Bw

\section{INTRODUCTION}

Neutrino-neutrino refraction can produce unexpected flavor oscillation phenomena in the form of self-induced flavor conversion in dense neutrino gases [1 39], for a review see Ref. [40]. One major complication is caused by the current-current nature of low-energy weak interactions. It implies that the refractive effect felt by a neutrino with velocity vector $\vec{v}$ caused by other neutrinos with velocity $\vec{v}^{\prime}$ is proportional to $1-\vec{v} \cdot \vec{v}^{\prime}$. If we study the flavor evolution of a homogeneous and isotropic neutrino gas (early universe) a seemingly obvious simplification is to integrate out the velocities, i.e., to drop the $\vec{v} \cdot \vec{v}^{\prime}$ term. The underlying assumption is that the solution of the equations of motion (EoM) will be homogeneous and isotropic as well. Moreover, it was tacitly assumed that unavoidable small deviations from these symmetries in the initial system will remain small in the solution as well. In the supernova ( $\mathrm{SN}$ ) context, the assumption of axially symmetric neutrino emission was used to integrate out the azimuth angle of neutrino propagation.

These universal assumptions go back to the earliest papers on the subject, but are nevertheless unjustified. Based on a linearized stability analysis in the context of axially symmetric SN neutrino streams, we have recently found a new class of solutions [41]. They spontaneously break axial symmetry and lead to azimuth-angle dependent flavor conversion effects. For simple neutrino spectra, the traditional bimodal instability occurs in IH, whereas the new multi-azimuth angle (MAA) instability occurs in NH. Meanwhile, numerical studies by other authors confirm the existence of this effect and its strong impact on collective flavor oscillations [42].

This change of paradigm calls into question numerous results in the previous literature. Any solution found under an uncontrolled symmetry assumption may have missed the dominant effect.

The mode analysis that has led to the discovery of the MAA instability is not complicated. The stability con- dition follows from an eigenvalue equation derived from the linearized EoMs. However, one finds stable or unstable conditions based on mathematical criteria that are not necessarily physically transparent or intuitive. We here provide a simple physical explanation in the simplest possible example that shows a new instability after a symmetry assumption has been relaxed.

Our system consists of two opposite moving beams 1 and 2 , each consisting of equal fluxes of $\nu_{e}$ and $\bar{\nu}_{e}$, all with the same energy. The modes 1 and 2 are taken to be identically prepared and therefore the solution should be symmetric under the exchange $1 \leftrightarrow 2$. We consider twoflavor oscillations with a small mixing angle. In IH one then finds the well-known bimodal instability [4, 5, 10] that leads to pendulumlike oscillations [12] between, say, $\nu_{e} \bar{\nu}_{e} \leftrightarrow \nu_{\tau} \bar{\nu}_{\tau}$. In $\mathrm{NH}$ the system is stable and the motion consists of small-amplitude harmonic oscillations.

After lifting the 1-2 symmetry assumption, the smallest disturbance could trigger a new instability. Indeed, one finds a new pendulumlike solution, now in NH, with the same flavor conversion effect. In IH, the new solution is stable. Unless the system is prepared exactly symmetric under $1 \leftrightarrow 2$ exchange, a pendulumlike solution appears in both hierarchies.

We describe the flavor content with polarization vectors $\mathbf{P}_{1,2}$ for neutrinos and $\overline{\mathbf{P}}_{1,2}$ for anti-neutrinos. ${ }^{12}$ In the limit of large neutrino-neutrino refraction, the traditional solution consists of all four polarization vectors sticking closely together and swinging in flavor space like

\footnotetext{
${ }^{1}$ We denote vectors in flavor space in bold-face characters, whereas vectors in coordinate space are denoted with an arrow.

2 Much of the literature uses the flavor-isospin convention where the polarization vectors describing neutrinos and anti-neutrinos of the same flavor point in opposite directions. In our convention they point in the same direction, allowing for a more straightforward visualization of self-induced flavor conversion, although in the equations we need to distinguish explicitly between $\nu$ and $\bar{\nu}$.
} 
an inverted plane pendulum. The new solution consists of $\mathbf{P}_{1}$ and $\overline{\mathbf{P}}_{1}$ to stick together as well as $\mathbf{P}_{2}$ and $\overline{\mathbf{P}}_{2}$, but these two pairs now pendulate in opposite directions in a single plane. This solution can arise only if initially the four vectors are not exactly aligned with each other.

It is actually simple to graphically understand these solutions and we begin, in Sec. III with a pictorial explanation. We then turn in Sec. III to a mathematical description, leading to two coupled anharmonic oscillators. We conclude in Sec. IV.

\section{PICTORIAL EXPLANATION}

To make contact with the earlier literature on the flavor pendulum [12] we use the same notation and describe the flavor content of the neutrino modes with polarization vectors in flavor space $\mathbf{P}$ and $\overline{\mathbf{P}}$, where overbarred quantities refer to antiparticles. If we consider $N$ momentum modes with momenta $\vec{p}_{i}(i=1, \ldots, N)$, the vacuum oscillation frequency of each mode is $\omega_{i}=\Delta m^{2} / 2 E_{i}$ and the velocity vectors are $\vec{v}_{i}=\vec{p}_{i} / E_{i}$. The oscillation equations for neutrino and antineutrino mode $j$ is

$$
\begin{aligned}
& \partial_{t} \mathbf{P}_{j}=\left[+\omega_{j} \mathbf{B}+\mu \sum_{i=1}^{N}\left(\mathbf{P}_{i}-\overline{\mathbf{P}}_{i}\right)\left(1-\vec{v}_{i} \cdot \vec{v}_{j}\right)\right] \times \mathbf{P}_{j}, \\
& \partial_{t} \overline{\mathbf{P}}_{j}=\left[-\omega_{j} \mathbf{B}+\mu \sum_{i=1}^{N}\left(\mathbf{P}_{i}-\overline{\mathbf{P}}_{i}\right)\left(1-\vec{v}_{i} \cdot \vec{v}_{j}\right)\right] \times \overline{\mathbf{P}}_{j},
\end{aligned}
$$

where we have ignored matter effects. Here $\mu \sim \sqrt{2} G_{\mathrm{F}} n_{\nu}$ is a measure of the neutrino-neutrino interaction energy in the dense neutrino gas.

For $\mu=0$ the evolution consists of a precession around the mass direction $\mathbf{B}$ in flavor space in opposite directions for neutrinos and anti-neutrinos (top-panel of Fig. 1). If neutrinos are prepared in flavor eigenstates the initial polarization vectors are tilted relative to $\mathbf{B}$ by twice the vacuum mixing angle. We choose the tilting direction to be in the $x-z$ plane in flavor space.

As a next step, we assume an isotropic ensemble and, following established practice, sum over all polarization vectors and drop the $1-\vec{v}_{i} \cdot \vec{v}_{j}$ factor. Of course, this is the crucial step of assuming that solutions of the EoMs will be isotropic because the system was prepared in such a state. In addition, we assume equal energies for all particles. Then the EoMs are simply $\dot{\mathbf{P}}=(\omega \mathbf{B}+\mu \mathbf{D}) \times \mathbf{P}$, where $\mathbf{D}=\mathbf{P}-\overline{\mathbf{P}}$, and negative $\omega$ for anti-neutrinos. The EoMs become even simpler when we introduce the variable $\mathbf{Q}=\mathbf{P}+\overline{\mathbf{P}}-(\omega / \mu) \mathbf{B}$ so that $\dot{\mathbf{Q}}=\mu \mathbf{D} \times \mathbf{Q}$ and $\dot{\mathbf{D}}=\omega \mathbf{B} \times \mathbf{Q}$. The EoM for $\mathbf{Q}$ involves a cross product so that the length of $\mathbf{Q}$ is conserved and one can show that its dynamics is that of a gyroscopic pendulum [12]. For exact particle-antiparticle symmetry the solution is even simpler. Taking initially $\mathbf{P}=\overline{\mathbf{P}}$ and thus $\mathbf{D}=0$, the motion of $\mathbf{Q}$ is that of a plane pendulum, representing the generic case of the bimodal instability.
In a pictorial explanation of this effect one begins with all polarization vectors initially aligned with the flavor direction. The flavor direction is taken to be nearly aligned with $\mathbf{B}$ up to the mismatch caused by the small mixingangle, i.e., we assume IH. The motion begins as pure vacuum oscillations (middle panel of Fig. 1). The opposite direction of precession drives $\mathbf{P}$ and $\overline{\mathbf{P}}$ apart and after a short while a non-zero vector $\mathbf{D}$ has developed. Assuming that $\mu \gg \omega$ and when $\mathbf{D}$ has become sufficiently long, the further evolution will be dominated by $\mu \mathbf{D}$ which points in the $y$ direction. So now both $\mathbf{P}$ and $\overline{\mathbf{P}}$ precess around $\mathbf{D}$ in a direction away from $\mathbf{B}$. This is the essence of the bimodal instability. Of course, one needs to go through the equations in detail to recognize that the full motion is that of an inverted pendulum.

One way of switching the neutrino mass hierarchy in these equations is $\Delta m^{2} \rightarrow-\Delta m^{2}$ or $\omega \rightarrow-\omega$. The polarization vectors now precess in the opposite directions
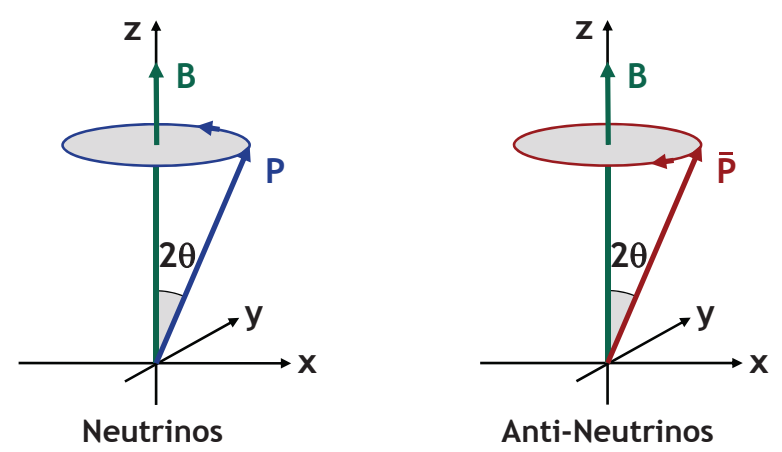

Anti-Neutrinos
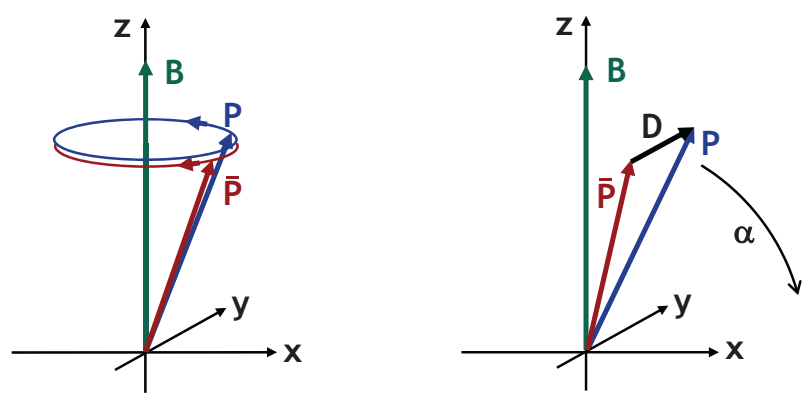

Inverted Hierarchy: Bimodal Instability

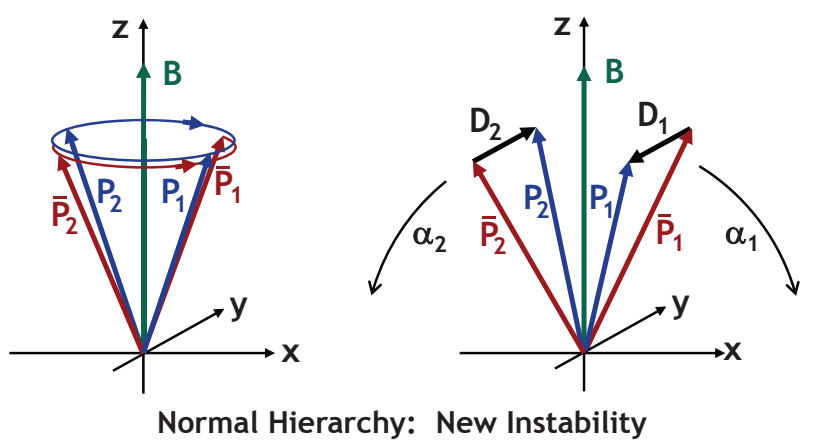

FIG. 1: Visualization of flavor oscillations and the bimodal and new instability as described in the text. 
around $\mathbf{B}$ so that the generated $\mathbf{D}$ points in the opposite direction. Now the precession around $\mathbf{D}$ has opposite sign and both $\mathbf{P}$ and $\overline{\mathbf{P}}$ move toward $\mathbf{B}$, not away from it. If the initial misalignment with $\mathbf{B}$ was small, the resulting motion is a small-amplitude harmonic swinging around the $\mathbf{B}$ direction.

Next we consider our two-beam example with opposite velocity vectors. The EoMs are now explicitly

$$
\begin{aligned}
& \partial_{t} \mathbf{P}_{1}=\left(+\omega \mathbf{B}+\mu \mathbf{D}_{2}\right) \times \mathbf{P}_{1}, \\
& \partial_{t} \overline{\mathbf{P}}_{1}=\left(-\omega \mathbf{B}+\mu \mathbf{D}_{2}\right) \times \overline{\mathbf{P}}_{1}, \\
& \partial_{t} \mathbf{P}_{2}=\left(+\omega \mathbf{B}+\mu \mathbf{D}_{1}\right) \times \mathbf{P}_{2}, \\
& \partial_{t} \overline{\mathbf{P}}_{2}=\left(-\omega \mathbf{B}+\mu \mathbf{D}_{1}\right) \times \overline{\mathbf{P}}_{2},
\end{aligned}
$$

where $\mathbf{D}_{i}=\mathbf{P}_{i}-\overline{\mathbf{P}}_{i}$ with $i=1$ or 2 . Assuming that the 1 and 2 modes evolve identically, we are back to the previous case of an isotropic system, except that here we have a reflection symmetry between 1 and 2 .

However, the two-beam system supports another class of solutions that obtain if we prepare the 1 and 2 modes initially tilted in opposite directions. We consider $\mathrm{NH}$ so that the initial vacuum precession is opposite to the previously unstable case. From the third panel in Fig. 1 one easily gleans the resulting directions of $\mathbf{D}_{1}$ and $\mathbf{D}_{2}$. The key point is that mode 1 feels the refractive effect caused by mode 2 , and the other way round. Therefore, the precession around the respective $\mathbf{D}_{1,2}$ vectors leads to motions away from $\mathbf{B}$ and subsequently to a pendulum with the 1 and 2 modes swinging in opposite directions. So this configuration is unstable in NH.

Switching the hierarchy back to IH reverses the directions of the $\mathbf{D}_{1,2}$ vectors and results in the familiar small-amplitude oscillation around the $\mathbf{B}$ direction. The anti-symmetric mode is unstable in the opposite hierarchy case compared with the bimodal instability.

Of course, if the system is prepared precisely such that initially all polarization vectors are the same, then only the traditional bimodal instability can occur. The smallest deviation from this exact initial symmetry causes an admixture of the anti-symmetric mode. The exponentially growing mode always wins. Therefore, any realistic initial condition provides a seed for a run-away solution in both hierarchies.

\section{COUPLED OSCILLATORS}

We now turn to a more formal analysis of the twobeam example. To this end we write the EoMs somewhat more symmetrically by introducing the sum vectors $\mathbf{S}_{i}=$ $\mathbf{P}_{i}+\overline{\mathbf{P}}_{i}$ with $i=1$ or 2 and find

$$
\begin{aligned}
\dot{\mathbf{S}}_{1} & =\mu \mathbf{D}_{2} \times \mathbf{S}_{1}+\omega \mathbf{B} \times \mathbf{D}_{1}, \\
\dot{\mathbf{S}}_{2} & =\mu \mathbf{D}_{1} \times \mathbf{S}_{2}+\omega \mathbf{B} \times \mathbf{D}_{2}, \\
\dot{\mathbf{D}}_{1} & =\omega \mathbf{B} \times \mathbf{S}_{1}+\mu \mathbf{D}_{2} \times \mathbf{D}_{1}, \\
\dot{\mathbf{D}}_{2} & =\omega \mathbf{B} \times \mathbf{S}_{2}+\mu \mathbf{D}_{1} \times \mathbf{D}_{2} .
\end{aligned}
$$

We simplify further as in the previous section and assume exact particle-antiparticle symmetry. We also assume that all polarization vectors are initially in the $x-z$ plane and that the corresponding particle and anti-particle vectors are prepared identically. The system then evolves symmetrically such that the $\mathbf{S}_{i}$ vectors move in the $x-z$ plane, whereas the $\mathbf{D}_{i}$ vectors are oriented along the $y$ direction. Therefore, the terms $\mathbf{D}_{1} \times \mathbf{D}_{2}$ drop out.

In analogy to the previous section, we can now easily identify two solutions that are symmetric or antisymmetric under $1 \leftrightarrow 2$. In the symmetric case, all polarization vectors are initially prepared identically. Then $\mathbf{D}_{2}=\mathbf{D}_{1}$ and we find two pairs of decoupled equations. Likewise, if the 1 and 2 modes are prepared with opposite $x$ components, we have $\mathbf{D}_{2}=-\mathbf{D}_{1}$. Therefore, the two eigenmodes correspond to equations of the form

$$
\begin{aligned}
\dot{\mathbf{S}} & = \pm \mu \mathbf{D} \times \mathbf{S}+\omega \mathbf{B} \times \mathbf{D}, \\
\dot{\mathbf{D}} & =\omega \mathbf{B} \times \mathbf{S} .
\end{aligned}
$$

Changing the sign of $\mu$, like changing the sign of $\omega$, corresponds to a change in hierarchy. Therefore, the solutions of one case in one hierarchy is identical to the solution of the other case with opposite hierarchy.

These pure symmetric or anti-symmetric solutions can each be brought to the form of a pendulum equation by introducing the vector $\mathbf{Q}=\mathbf{S} \mp(\omega / \mu) \mathbf{B}$. The EoMs are then $\dot{\mathbf{Q}}= \pm \mu \mathbf{D} \times \mathbf{Q}$ and $\dot{\mathbf{D}}=\omega \mathbf{B} \times \mathbf{Q}$. However, the vector $\mathbf{Q}$ is defined differently for the two cases. Still, if we go to the limit $\mu \gg \omega$ we can approximately equal $\mathbf{S}$ with $\mathbf{Q}$ and the motion of each $\mathbf{S}_{i}$ is approximately a circle in the $x-z$ plane with its center at the origin. We thus seek solutions of the form

$$
\mathbf{S}_{i}(t)=S_{i}\left(\begin{array}{c}
\sin \alpha_{i}(t) \\
0 \\
\cos \alpha_{i}(t)
\end{array}\right) \quad \text { and } \quad \mathbf{D}_{i}(t)=\left(\begin{array}{c}
0 \\
D_{i}(t) \\
0
\end{array}\right)
$$

leading to $\dot{\alpha}_{1}=\mu D_{2}, \dot{\alpha}_{2}=\mu D_{1}$, and $\dot{D}_{i}=\omega S_{i} \sin \alpha_{i}$. Taking the second derivative then leads to the oscillator equations $\ddot{\alpha}_{1}=\omega \mu S_{2} \sin \alpha_{2}$ and $\ddot{\alpha}_{2}=\omega \mu S_{1} \sin \alpha_{1}$. With $S=S_{1}=S_{2}$ and $\kappa^{2}=\omega \mu S$ we finally have

$$
\begin{aligned}
& \ddot{\alpha}_{1}=\kappa^{2} \sin \alpha_{2}, \\
& \ddot{\alpha}_{2}=\kappa^{2} \sin \alpha_{1} .
\end{aligned}
$$

Our simplified system is therefore equivalent to two maximally mixed anharmonic oscillators.

The eigenmodes of this system consist of a symmetric solution with $\alpha_{1}(t)=\alpha_{2}(t)$ and an anti-symmetric one with $\alpha_{1}(t)=-\alpha_{2}(t)$. In the former case, with $\alpha=\alpha_{1}=\alpha_{2}$, the EoMs reduce to the single equation $\ddot{\alpha}=\kappa^{2} \sin \alpha$. We always assume that initially $\mathbf{D}_{i}=0$, i.e., the particle and anti-particle modes begin in the same flavor state so that $\dot{\alpha}(0)=0$. If the initial angle is small, $\alpha(0) \ll 1$, the symmetric solution corresponds to an inverted pendulum, i.e., an anharmonic oscillator beginning near the maximum of the potential. Switching the hierarchy amounts to $\omega \rightarrow-\omega$ and thus $\kappa^{2} \rightarrow-\kappa^{2}$. In this case, the symmetric solution corresponds to a harmonic oscillator with a small initial amplitude. 

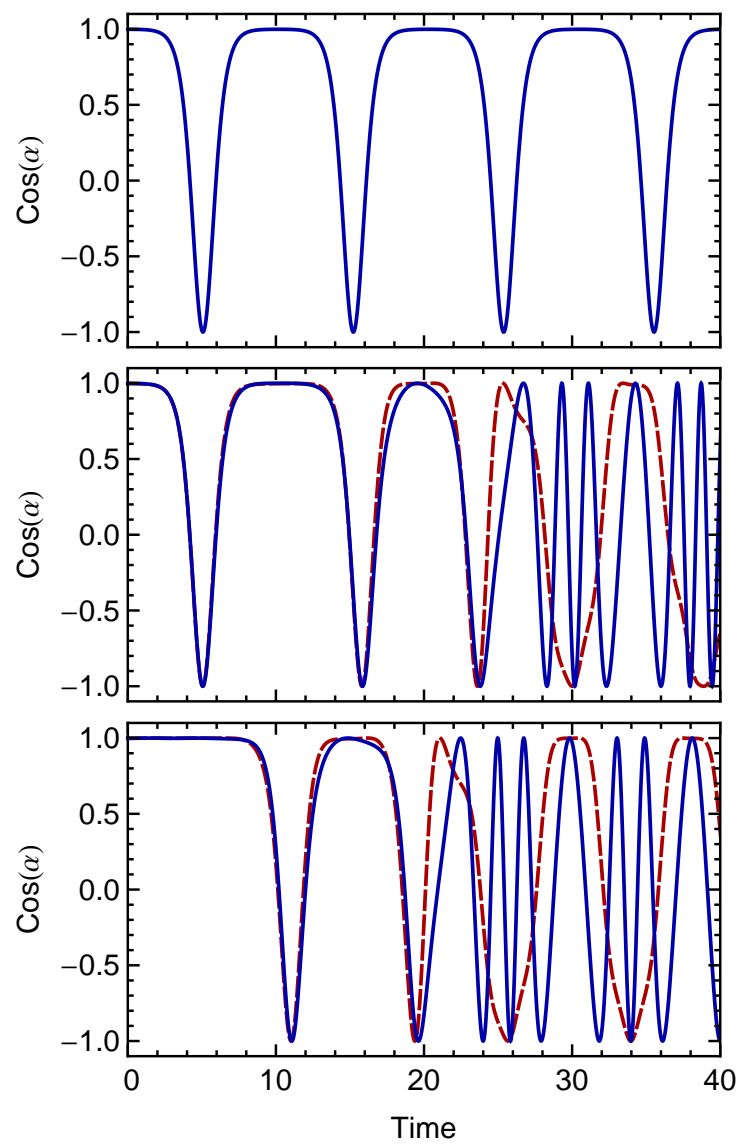

FIG. 2: Solution for $\alpha_{1}$ (blue solid line) and $\alpha_{2}$ (red dashed line) with $\kappa=1$ and $\alpha_{1}(0)=\alpha_{2}(0)=0.05$. Top: Bimodal solution in IH. Middle: Same with $1 \%$ difference in $\kappa^{2}$ in the two EoMs. Bottom: Same in NH, showing the new instability.

For the anti-symmetric solution we write $\alpha=\alpha_{1}=$ $-\alpha_{2}$ so that the EoMs reduce to $\ddot{\alpha}=-\kappa^{2} \sin \alpha$. Therefore, the original case of IH now corresponds to the smallamplitude harmonic oscillator. Switching the hierarchy changes $\kappa^{2} \rightarrow-\kappa^{2}$ and we are back to the inverted pendulum. In other words, switching the hierarchy and switching between the symmetric and anti-symmetric solution each causes a sign change in $\ddot{\alpha}= \pm \kappa^{2} \sin \alpha$.

We may prepare the system initially such that both modes have exactly equal particle densities in exact flavor eigenstates. In this case $\alpha_{1}(0)=\alpha_{2}(0)$ and only the symmetric mode is excited. This is the traditional case where the solution was assumed to inherit the symmetry of the initial condition and small deviations from that symmetry were assumed to remain small.

However, the latter assumption is unjustified in the case of $\mathrm{NH}$ where the traditional solution is stable, whereas the anti-symmetric solution is unstable. The smallest deviation of the initial state from perfect symmetry provides a seed for the exponentially growing solution which then eventually dominates.

We illustrate this behavior with a simple example in
Fig. 2 We use $\kappa=1$ and the initial angles $\alpha_{1}(0)=$ $\alpha_{2}(0)=0.05$. In IH we then find the usual bimodal solution with pendulumlike periodic flavor conversion (top panel). To disturb the perfect initial symmetry we can either make the initial angles slightly different or assume slightly different particle fluxes in the two beams. We choose the latter approach, implying that $S_{1}$ is slightly different from $S_{2}$, i.e., $\kappa^{2}$ in the two equations is a bit different. We use a $1 \%$ difference. In this case the bimodal solution at first proceeds as before, but it is now coupled to the harmonic oscillator of the anti-symmetric solution. Therefore, after a few pendulum dips, $\alpha_{1}(t)$ and $\alpha_{2}(t)$ get out of step, resulting in chaotic behavior.

Switching the hierarchy (bottom panel) would provide the traditional stable solution if the initial conditions were perfectly symmetric. Implementing our small mismatch has at first no effect, but the unstable admixture of the anti-symmetric mode quickly grows enough to take over and again we get pendulumlike behavior that also turns chaotic.

Even for exact initial symmetry, numerical noise is enough to trigger the run-away solution in $\mathrm{NH}$, at least when using Mathematica to solve the equations with standard settings. In our example, the first dip triggered by numerical noise was roughly at $t=45$.

Our example was constructed to be perfectly symmetric between particles and antiparticles, restricting the motion to the $x-z$ plane and we have assumed $\mu \gg \omega$. Numerical examples solving the full EoMs of Eq. (3) in various cases show analogous effects. In other words, if we relax further symmetry assumptions does not prevent the new instability, but also does not introduce yet other qualitatively new effects. From a linearized stability analysis of our general two-beam system we indeed find exactly two instabilities. Of course, in the general case the instabilities only arise for certain ranges of $\mu$ values.

\section{CONCLUSION}

We have constructed the simplest example that graphically illustrates the recent insight that the multi-angle nature of neutrino-neutrino refraction can lead to instabilities that spontaneously break the symmetry of initial conditions. In particular, we have found that the traditional flavor pendulum actually occurs in both hierarchies if one allows angular modes to evolve independently.

When the neutrino and anti-neutrino populations are not identical, the more realistic case in situations of practical interest, the system is stable at large densities. It remains to be seen if, when slowly reducing the density, one can obtain ordered solutions and spectral features such as spectral splits. Likewise, one can ask for the existence of pure precession modes, their stability, and many other properties.

Our simple example was meant as a proxy for the somewhat confusing multi-azimuth angle (MAA) instability 
that occurs in axially symmetric SN neutrino fluxes. It was found that this instability occurs even when using only two discrete azimuth angles, i.e., for a two-beam example. In the SN case, these two modes intersect at a small angle, but have opposite directions in the transverse plane. As the magnitude of the intersection angle only influences the effective neutrino-neutrino interaction strength, our two-beam example closely mimics this case. The formal stability analysis reveals that there is only one additional instability, not one for every discrete azimuth direction. The traditional bimodal instability has no $\varphi$ dependence, the new instability a dipole structure in $\varphi$.

Relaxing unjustified symmetry assumptions concerning the solutions of the equations of collective flavor oscillations leads to new effects. What this means in practice, for example in the context of SN neutrino oscillations, remains to be explored. It is certain, however, that one cannot trust any result that relies on symmetries that may get spontaneously broken by the interacting neutrino system.

\section{Acknowledgments}

This work was partly supported by the Deutsche Forschungsgemeinschaft under grant EXC-153 (Cluster of Excellence "Origin and Structure of the universe") and by the European Union under grant PITNGA-2011-289442 (FP7 Initial Training Network "Invisibles"). D. S. acknowledges support by the Fundação para a Ciência e Tecnologia (Portugal) under grant SFRH/BD/66264/2009.
[1] J. Pantaleone, Phys. Lett. B 287, 128 (1992).

[2] G. Sigl and G. Raffelt, Nucl. Phys. B 406, 423 (1993).

[3] S. Samuel, Phys. Rev. D 48, 1462 (1993).

[4] V. A. Kostelecky and S. Samuel, Phys. Lett. B 318, 127 (1993).

[5] S. Samuel, Phys. Rev. D 53, 5382 (1996).

[6] J. T. Pantaleone, Phys. Rev. D 58, 073002 (1998).

[7] A. D. Dolgov et al., Nucl. Phys. B 632, 363 (2002).

[8] A. Friedland and C. Lunardini, Phys. Rev. D 68, 013007 (2003).

[9] R. F. Sawyer, Phys. Rev. D 72, 045003 (2005).

[10] H. Duan, G. M. Fuller and Y.-Z. Qian, Phys. Rev. D 74, 123004 (2006).

[11] H. Duan, G. M. Fuller, J. Carlson and Y.-Z. Qian, Phys. Rev. D 74, 105014 (2006).

[12] S. Hannestad, G. Raffelt, G. Sigl and Y. Y. Y. Wong, Phys. Rev. D 74, 105010 (2006); Erratum ibid. 76, 029901 (2007).

[13] A. Friedland, B. H. J. McKellar and I. Okuniewicz, Phys. Rev. D 73, 093002 (2006).

[14] G. G. Raffelt and G. Sigl, Phys. Rev. D 75, 083002 (2007).

[15] G. Raffelt and A. Yu. Smirnov, Phys. Rev. D 76, 081301 (2007); 77, 029903(E) (2008); Phys. Rev. D 76, 125008 (2007).

[16] H. Duan, G. M. Fuller and Y.-Z. Qian, Phys. Rev. D 76, 085013 (2007).

[17] G. L. Fogli, E. Lisi, A. Marrone and A. Mirizzi, JCAP 12 (2007) 010.

[18] G. L. Fogli, E. Lisi, A. Marrone, A. Mirizzi and I. Tamborra, Phys. Rev. D 78, 097301 (2008).

[19] R. F. Sawyer, Phys. Rev. D 79, 105003 (2009).

[20] G. G. Raffelt, Phys. Rev. D 78, 125015 (2008).

[21] A. Esteban-Pretel, A. Mirizzi, S. Pastor, R. Tomàs, G. G. Raffelt, P. D. Serpico and G. Sigl, Phys. Rev. D 78, 085012 (2008).

[22] B. Dasgupta, A. Dighe, G. Raffelt and A. Yu. Smirnov, Phys. Rev. Lett. 103, 051105 (2009).
[23] G. G. Raffelt and I. Tamborra, Phys. Rev. D 82, 125004 (2010).

[24] R. F. Sawyer, Phys. Rev. D 83, 065023 (2011).

[25] H. Duan and A. Friedland, Phys. Rev. Lett. 106, 091101 (2011).

[26] A. Banerjee, A. Dighe and G. Raffelt, Phys. Rev. D 84, 053013 (2011).

[27] S. Sarikas, G. G. Raffelt, L. Hüdepohl and H.-T. Janka, Phys. Rev. Lett. 108, 061101 (2012).

[28] G. G. Raffelt, Phys. Rev. D 83, 105022 (2011).

[29] Y. Pehlivan, A. B. Balantekin, T. Kajino and T. Yoshida, Phys. Rev. D 84, 065008 (2011).

[30] M. Dvornikov, Nucl. Phys. B 855, 760 (2012).

[31] S. Chakraborty, T. Fischer, A. Mirizzi, N. Saviano and R. Tomàs, Phys. Rev. D 84, 025002 (2011); Phys. Rev. Lett. 107, 151101 (2011).

[32] N. Saviano, S. Chakraborty, T. Fischer and A. Mirizzi, Phys. Rev. D 85, 113002 (2012).

[33] A. Mirizzi and P. D. Serpico, Phys. Rev. D 86, 085010 (2012).

[34] S. Sarikas, D. de Sousa Seixas and G. Raffelt, Phys. Rev. D 86, 125020 (2012).

[35] J. F. Cherry, J. Carlson, A. Friedland, G. M. Fuller and A. Vlasenko, Phys. Rev. Lett. 108, 261104 (2012).

[36] S. Sarikas, I. Tamborra, G. Raffelt, L. Hüdepohl and H.-T. Janka, Phys. Rev. D 85, 113007 (2012).

[37] A. de Gouvea and S. Shalgar, JCAP 1210, 027 (2012); JCAP 1304, 018 (2013).

[38] C. Volpe, D. Väänänen and C. Espinoza, Phys. Rev. D 87, 113010 (2013).

[39] J. F. Cherry, J. Carlson, A. Friedland, G. M. Fuller and A. Vlasenko, Phys. Rev. D 87, 085037 (2013).

[40] H. Duan, G. M. Fuller and Y.-Z. Qian, Annu. Rev. Nucl. Part. Sci. 60, 569 (2010).

[41] G. Raffelt, S. Sarikas and D. de Sousa Seixas, Phys. Rev. Lett. in press (2013) arXiv:1305.7140.

[42] A. Mirizzi, arXiv:1308.1402 